\title{
Impact of the mechanism of mitral regurgitation on clinical outcomes in patients after mitral
} valve surgery

\author{
Jolanta Rzucidło-Resil'1, Dariusz Plicner2,3, Andrzej Gackowski1,4, Bogusław Kapelak²,4, Jarosław Stoliński² \\ 1 Noninvasive Cardiovascular Laboratory, John Paul II Hospital, Kraków, Poland \\ 2 Department of Cardiac Surgery and Transplantology, John Paul II Hospital, Kraków, Poland \\ 3 Faculty of Medicine and Health Sciences, Andrzej Frycz Modrzewski Krakow University, Kraków, Poland \\ 4 Department of Coronary Disease and Heart Failure, Institute of Cardiology, John Paul II Hospital, Kraków, Poland
}

\section{KEY WORDS}

mitral regurgitation, mitral valve surgery, etiology, outcomes

\section{EDITORIAL}

page 505

\section{ABSTRACT}

BACKGROUND Mitral regurgitation (MR) is the second most frequent indication for valve surgery. There are few studies addressing mitral valve (MV) surgery in the context of etiology of MR.

AIMS We aimed to compare postoperative outcomes in the context of the etiological mechanism of MR in patients after MV surgery.

METHODS The study group included 337 consecutive patients with severe MR. Preoperative comorbidities, postoperative clinical course, and predictors of in-hospital mortality were assessed.

RESULTS Primary etiology of MR was observed in $72 \%$ of patients, and of secondary, in $28 \%(P<0.001)$. Among the primary MR group, the most common etiological factor was fibroelastic deficiency (79\%), followed by Barlow disease $(16 \%)$ and rheumatic disease $(5 \%)(P<0.001)$. Secondary MR was seen in ischemic heart disease $(67 \%)$ and dilated cardiomyopathy $(33 \%)(P<0.001)$. The incidence of death and complications following surgery did not differ between the groups. Univariate analysis revealed that higher risk of death was associated with older age, severe heart failure symptoms, impaired left ventricular ejection fraction, previous percutaneous coronary interventions, cardiopulmonary bypass time, low cardiac output syndrome, and wound infections $(P=0.004, P<0.001, P=0.005, P=0.009, P=0.002$, $P=0.006$, and $P=0.03$, respectively). Also MV replacement with concomitant other valve surgery increased the risk of mortality $(P=0.049)$.

CONCLUSIONS This study indicates that the clinical outcomes and in-hospital mortality in patients with severe MR correlate with the type of procedure and concomitant perioperative comorbidities rather than the etiological mechanism of MR itself.

\footnotetext{
Correspondence to:

Dariusz Plicner, MD, PhD, Department of Cardiosurgery, John Paul II Hospital, ul. Prądnicka 80, 31-202 Kraków, Poland, phone: +48 126143075 , email: plicner.dariusz@gmail.com Received: February 4, 2019. Revision accepted: March 1, 2019 Published online: March 5, 2019. Kardiol Pol. 2019; 77 (5): 525-534 doi:10.5603/KP.a2019.0043 Copyright by Polskie Towarzystwo Kardiologiczne, Warszawa 2019
}

INTRODUCTION Mitral regurgitation (MR) is the second most frequent indication for valve surgery and affects about $2 \%$ of the total population. ${ }^{1,2}$ It is essential to distinguish the cause of MR, particularly in relation to disease management. The most common classification divides $\mathrm{MR}$ according to its mechanism into primary and secondary forms. In primary MR, 1 or more components of the valve apparatus are affected as a result of mitral valve (MV) degeneration, including rheumatic heart disease, fibroelastic deficiency, or Barlow disease. Endocarditis is one reason for primary MR that is specifically discussed in the European Society of Cardiology and European Association for Cardio-Thoracic Surgery guidelines. ${ }^{1,3}$ In secondary MR, the valve apparatus is anatomically intact and MR results from a disproportion between closing and tethering forces on the valve secondary to left ventricular dilation and dysfunction. It is most frequently seen in dilated or ischemic cardiomyopathies..$^{1,3}$ 


\section{WHAT'S NEW}

Appropriate estimation of the etiology of mitral regurgitation (MR) is a crucial part of adequate disease management. No previous studies have compared postoperative outcomes depending on the etiological mechanism of MR in consecutive patients after mitral valve surgery. This study was conducted in the real-life setting of a cardiosurgical unit and enrolled unselected consecutive patients. We found that the etiological mechanism of MR did not correlate with the postoperative clinical course. In-hospital mortality correlated with the type of procedure and associated comorbidities rather than the etiological mechanism of MR itself. Patients with severe MR and associated comorbidities require a thorough assessment and attention to additional risk factors to reduce the risk of perioperative complications and death.

Mitral valve surgery is considered the gold standard treatment for patients with severe symptomatic MR or with severe asymptomatic MR and left ventricular dysfunction or dilation. ${ }^{1}$ There are limited studies addressing MV surgery in the context of the etiology of MR.4-8 Neither European System for Cardiac Operative Risk Evaluation (EuroSCORE) II nor the Society of Thoracic Surgeons (STS) score incorporated data regarding MR mechanism into their predictive model. Risk scores have major limitations for practical use in this setting because they do not take disease severity into sufficient consideration, and they do not include major risk factors such as frailty, porcelain aorta, chest radiation as well as echocardiographic parameters (eg, right ventricular function). ${ }^{1}$

To the best of our knowledge, no previous studies have compared postoperative outcomes as well as in-hospital mortality rate in the context of etiological mechanism of MR in consecutive patients after MV surgery. This prompted us to assess the impact of etiology of MR on clinical outcomes in the early postoperative period after MV surgery. In this study, we report our experience with MV surgery in consecutive patients with different types of primary and secondary MR. We analyzed the etiology, risk factors, and clinical outcomes of these patients.

METHODS Patients The investigation conforms to the principles outlined in the Declaration of Helsinki. A total of 337 consecutive patients with severe MR were enrolled in the study from January 2015 to December 2017. Patients who required concomitant tricuspid annuloplasty, aortic valve replacement, or coronary artery bypass grafting (CABG) for coronary artery disease were included in the study. Exclusion criteria were as follows: patients requiring an emergency or urgent surgery due to MR caused by mechanical complication of myocardial infarction (rupture of the papillary muscle), endocarditis or prosthesis dysfunction (paravalvular leaks, prosthetic valve thrombosis), and left ventricular ejection fraction (LVEF) of less than $30 \%$.
The diagnosis of MR was based on preoperative echocardiography and confirmed by the surgeon's direct assessment of the valve., ${ }^{1,9}$ Two-dimensional Doppler transthoracic echocardiography was performed, using an iE33/ EPIQ 7 (Philips Healthcare, Andover, Massachusetts, United States). Additionally, 3-dimensional transesophageal echocardiography was performed in all patients before surgery, using the same ultrasound system. Surgery was performed in both symptomatic and asymptomatic patients who met the echocardiographic criteria for severe MR. Intraoperative transesophageal echocardiography was performed in all patients who underwent MV repair, and transthoracic echocardiography was performed in all patients before discharge.

Demographic and clinical data, including the EuroSCORE II and the STS score, were collected prospectively. Low cardiac output (LCO) was diagnosed if the patient required intra-aortic balloon pump or inotropic therapy to maintain the systolic blood pressure of more than $90 \mathrm{~mm} \mathrm{Hg}$ and cardiac output of more than $2.2 \mathrm{l} / \mathrm{min} / \mathrm{m}^{2}$ for 30 minutes or more after correcting abnormalities of all electrolyte and blood gases and after adjusting the preload and afterload to its optimal value. Patients who received a low dose of dopamine $(\leq 3 \mu \mathrm{g} / \mathrm{kg} / \mathrm{min})$ and those who required vasoconstricting medications to increase low peripheral vascular resistance in the presence of high cardiac output $\left(\geq 2.5 \mathrm{l} / \mathrm{min} / \mathrm{m}^{2}\right)$ were not considered to have LCO. ${ }^{10,11}$

New postoperative atrial fibrillation was diagnosed as atrial fibrillation or flutter that occurred during the postoperative period before hospital discharge and required treatment. This did not include transient, nonsustained arrhythmias or arrhythmias treated only with magnesium or potassium supplementation. ${ }^{12}$

In-hospital cardiovascular death was defined as death occurring during the same hospitalization period as the MV surgery.

Surgical technique The MV procedures were performed through a median sternotomy or right or left minithoracotomy.

Conventional mitral surgery was performed through a median sternotomy. Heparin (500 $\mathrm{IU} / \mathrm{kg}$ ) was administered as anticoagulant therapy before the start of cardiopulmonary bypass (CPB) and was monitored by means of the activated clotting time, which had to be above 400 seconds during $\mathrm{CPB}$. All operations were performed on $\mathrm{CPB}$, consisting of a nonpulsatile roller pump (Jostra Medizintechnik AG, Hirrlingen, Germany) and an in-line arterial blood filter (Jostra Medizintechnik AG) under moderate systemic hypothermia (esophageal temperature, $32^{\circ} \mathrm{C}$ ). Mean arterial pressure was maintained between 40 and $60 \mathrm{~mm} \mathrm{Hg}$ and CPB blood flow 
TABLE 1 Baseline characteristics of patients with primary and secondary mitral regurgitation

\begin{tabular}{|c|c|c|c|c|c|}
\hline \multicolumn{2}{|l|}{ Variable } & $\begin{array}{l}\text { All patients } \\
(\mathrm{n}=337)\end{array}$ & $\begin{array}{l}\text { Primary } \\
\quad \text { MR } \\
(n=243)\end{array}$ & $\begin{array}{l}\text { Secondary } \\
\text { MR } \\
(n=94)\end{array}$ & Pvalue \\
\hline \multicolumn{2}{|l|}{ Age, y } & $65(60.5-75)$ & $64(59-70)$ & $69(63-74.8)$ & $<0.001$ \\
\hline \multicolumn{2}{|c|}{ Male sex, n (\%) } & $224(66.5)$ & $163(67.1)$ & $61(64.9)$ & 0.80 \\
\hline \multirow{5}{*}{$\begin{array}{l}\text { Mechanism } \\
\text { of MR, } \\
\mathrm{n}(\%)\end{array}$} & $\begin{array}{l}\text { Rheumatic heart } \\
\text { disease }\end{array}$ & $12(3.6)$ & $12(4.9)$ & - & \multirow[t]{3}{*}{$<0.001$} \\
\hline & $\begin{array}{l}\text { Fibroelastic } \\
\text { deficiency }\end{array}$ & $192(57)$ & $192(79)$ & - & \\
\hline & Barlow disease & $39(11.6)$ & $39(16)$ & - & \\
\hline & $\begin{array}{l}\text { Ischemic heart } \\
\text { disease }\end{array}$ & $63(18.7)$ & - & $63(67)$ & \multirow[t]{2}{*}{$<0.001$} \\
\hline & $\begin{array}{l}\text { Dilated } \\
\text { cardiomyopathy }\end{array}$ & $31(9.2)$ & - & $31(33)$ & \\
\hline \multicolumn{2}{|l|}{$\mathrm{BSA}, \mathrm{m}^{2}$} & $1.9(1.7-2)$ & $1.9(1.7-2)$ & $1.9(1.8-2)$ & 0.57 \\
\hline \multicolumn{2}{|l|}{$\mathrm{BMI}, \mathrm{kg} / \mathrm{m}^{2}$} & $\begin{array}{l}26.4 \\
(24.2-29.6)\end{array}$ & $\begin{array}{l}26.2 \\
(24.3-29.6)\end{array}$ & $\begin{array}{l}27.5 \\
(24-29.6)\end{array}$ & 0.21 \\
\hline \multirow{2}{*}{$\begin{array}{l}\text { NYHA class, } \\
\mathrm{n}(\%)\end{array}$} & $\mathrm{I} / \mathrm{II}$ & $206(61.1)$ & $160(65.8)$ & $46(48.9)$ & \multirow[t]{2}{*}{0.006} \\
\hline & III/IV & $131(38.9)$ & $83(34.2)$ & $48(51.1)$ & \\
\hline \multicolumn{2}{|c|}{ Previous stroke, n (\%) } & $32(9.5)$ & $23(9.5)$ & $9(9.6)$ & 1.0 \\
\hline \multicolumn{2}{|c|}{ Diabetes mellitus, n (\%) } & $64(19)$ & $39(16)$ & $25(26.6)$ & 0.04 \\
\hline \multicolumn{2}{|c|}{ Hypertension, n (\%) } & $274(81.3)$ & $194(79.8)$ & $80(85.1)$ & 0.34 \\
\hline \multicolumn{2}{|c|}{ Thyroid disease, n (\%) } & $54(16)$ & $32(13.2)$ & $22(23.4)$ & 0.07 \\
\hline \multicolumn{2}{|c|}{ Preoperative AF, n (\%) } & $180(53.4)$ & $124(51)$ & $56(59.6)$ & 0.20 \\
\hline \multicolumn{2}{|c|}{$\begin{array}{l}\text { Preoperative pacemaker } \\
\text { implantation, } \mathrm{n}(\%)\end{array}$} & $19(5.6)$ & $8(3.3)$ & $11(11.7)$ & 0.006 \\
\hline \multicolumn{2}{|l|}{ LVEF, \% } & $\begin{array}{l}58 \\
(46-65)\end{array}$ & $\begin{array}{l}60 \\
(50-65)\end{array}$ & $\begin{array}{l}42.5 \\
(30.5-55)\end{array}$ & $<0.001$ \\
\hline \multicolumn{2}{|c|}{ Previous PCI, n (\%) } & $68(20.2)$ & $35(14.4)$ & $33(35.1)$ & $<0.001$ \\
\hline \multicolumn{2}{|l|}{ COPD, n (\%) } & $22(6.5)$ & $14(5.8)$ & $8(8.5)$ & 0.50 \\
\hline \multicolumn{2}{|c|}{ Renal failure, n (\%) } & $6(1.8)$ & $4(1.6)$ & $2(2.1)$ & 0.67 \\
\hline \multicolumn{2}{|c|}{ EuroSCORE II, points } & $1.7(1-3.3)$ & $1.4(0.9-2.7)$ & $2.9(1.6-5.3)$ & $<0.001$ \\
\hline \multicolumn{2}{|c|}{ STS score, points } & $1.4(0.7-2.4)$ & $1.2(0.6-1.9)$ & $2.1(1.2-3.6)$ & $<0.001$ \\
\hline
\end{tabular}

Data are presented as median (interquartile range) unless otherwise indicated.

Abbreviations: AF, atrial fibrillation; BMI, body mass index; $B S A$, body surface area; COPD, chronic obstructive pulmonary disease; EurOSCORE, European System for Cardiac Operative Risk Evaluation; LVEF, left ventricular ejection fraction; MR, mitral regurgitation; NYHA, New York Heart Association functional classification; PCI, percutaneous coronary interventions; STS, Society of Thoracic Surgeons

was maintained at 2.0 to $2.4 \mathrm{l} / \mathrm{min} / \mathrm{m}^{2}$. Besides $\mathrm{CO}_{2}$ insufflation, antegrade warm blood or cold crystalloid cardioplegia was applied, and MV surgery was performed using standard techniques.

For minimally invasive mitral surgery, tracheal intubation was performed with a double lumen tracheal tube. Anticoagulation was accomplished similarly as in the conventional mitral procedure. Vacuum-assisted $\mathrm{CPB}$ was instituted by peripheral cannulation of the femoral vessels (Bio-medicus Medtronic, Minneapolis, Unites States, and Quick
Draw Edwards Lifesciences, Irvine, California, United States). Both cannulas were placed under transesophageal echocardiography control. An approximate 5-cm transverse incision was made under the right nipple, and the chest was entered through the forth intercostal space on the right side. $\mathrm{CO}_{2}$ insufflation into the right hemithorax was performed. An aortic transthoracic cross-clamp was introduced through the third intercostal space, and antegrade cardioplegia was initiated by $2000 \mathrm{ml}$ of Custodiol (Dr. Franz Köhler Chemie, Alsbach-Hähnlein, Germany). Visualization of the MV was achieved through conventional left atrial incision in the interatrial groove. The surgery was performed using long-shafted instruments designed for a minimally invasive thoracoscopic surgery.

For the Harpoon procedure, that is, beating heart MV repair procedure, a small transverse incision was made under the left nipple and the device was inserted into the left ventricle. The entire procedure was guided by transesophageal echocardiography. Polytetrafluoroethylene cords were implanted into the posterior leaflet to restore the leaflet coaptation. The procedure was performed as described by Gammie et al. ${ }^{13}$

Patients from all groups received uniform postoperative care.

Statistical analysis Categorical variables were expressed as numbers and percentages. Quantitative variables were expressed as median and interquartile range (IQR). The null hypothesis of no differences between groups was tested using the Mann-Whitney test. For categorical variables, significance of differences between groups was assessed using the $\mathrm{X}^{2}$ test or Fisher test. A $P$ value of less than 0.05 was considered significant. No adjustment for multiple comparisons was made. All statistical analyses were performed using the $\mathrm{R}$ software, version 3.4 (The R Foundation for Statistical Computing, Vienna, Austria).

RESULTS Baseline characteristics The baseline characteristics of MR patients are shown in TABLES1 and 2. A group of 337 patients consisting of mostly hypertensive and overweight men with preoperative atrial fibrillation were studied. The etiology of MR was primary in $72 \%$ of patients and secondary in $28 \%(P<0.001)$. Patients with primary MR were younger than those with secondary MR $(P<0.001)$. Severe heart failure symptoms (New York Heart Association functional classification, class III or IV) occurred in $39 \%$ of patients and were more frequent in patients with secondary MR $(P=0.006)$. Furthermore, LVEF was much lower in this group $(P<0.001)$. Patients with secondary MR also had a higher incidence of diabetes mellitus, previous pacemaker implantations, and percutaneous coronary interventions 
TABLE 2 Baseline characteristics of patients according to etiology of mitral regurgitation

\begin{tabular}{|c|c|c|c|c|c|c|c|c|}
\hline \multirow{2}{*}{\multicolumn{2}{|c|}{ Variable }} & \multicolumn{3}{|c|}{ Primary MR $(n=243)$} & \multirow[t]{2}{*}{$P$ value } & \multicolumn{2}{|c|}{ Secondary MR $(n=94)$} & \multirow[t]{2}{*}{$P$ value } \\
\hline & & $\begin{array}{l}\text { Rheumatic } \\
\text { heart disease } \\
(n=12)\end{array}$ & $\begin{array}{l}\text { Fibroelastic } \\
\text { deficiency } \\
(n=192)\end{array}$ & $\begin{array}{l}\text { Barlow } \\
\text { disease } \\
(n=39)\end{array}$ & & $\begin{array}{l}\text { Ischemic heart } \\
\text { disease } \\
(n=63)\end{array}$ & $\begin{array}{l}\text { Dilated } \\
\text { cardiomyopathy } \\
(\mathrm{n}=31)\end{array}$ & \\
\hline \multicolumn{2}{|l|}{ Age, y } & $68.5(64.8-70)$ & $65(60-70)$ & $61(47-67)$ & $\begin{array}{l}0.003^{a} \\
0.001^{b}\end{array}$ & $69(62-75.5)$ & $69(63.5-73)$ & 0.99 \\
\hline \multicolumn{2}{|l|}{ Men, n (\%) } & $6(50)$ & $132(68.7)$ & $25(64.1)$ & 0.144 & $45(71.4)$ & $16(51.6)$ & 0.01 \\
\hline \multicolumn{2}{|l|}{$\mathrm{BSA}, \mathrm{m}^{2}$} & $1.8(1.8-1.9)$ & $1.9(1.7-2)$ & $1.9(1.7-2)$ & 0.859 & $1.9(1.8-2)$ & $1.9(1.8-2)$ & 0.55 \\
\hline \multicolumn{2}{|l|}{ BMI, $\mathrm{kg} / \mathrm{m}^{2}$} & $29.1(24.6-30.7)$ & $26.3(24.5-29.4)$ & $25.5(23.2-27)$ & 0.184 & $27.6(24.5-29.1)$ & $26.8(23.3-31.2)$ & 0.65 \\
\hline \multirow[t]{2}{*}{ NYHA class, n (\%) } & $\mathrm{I} / \mathrm{II}$ & $7(58.3)$ & $126(65.6)$ & $27(69.2)$ & \multirow[t]{2}{*}{0.541} & $25(39.7)$ & $21(67.7)$ & \multirow[t]{2}{*}{0.02} \\
\hline & III/IV & $5(41.7)$ & $66(34.4)$ & $12(30.8)$ & & $38(60.3)$ & $10(32.3)$ & \\
\hline \multicolumn{2}{|c|}{ Previous stroke, n (\%) } & $2(16.7)$ & $19(9.9)$ & $2(5.1)$ & 0.096 & $5(7.9)$ & $4(12.9)$ & 0.47 \\
\hline \multicolumn{2}{|c|}{ Diabetes mellitus, n (\%) } & $2(16.7)$ & $33(17.2)$ & $4(10.2)$ & 0.271 & $16(25.4)$ & $9(29)$ & 0.90 \\
\hline \multicolumn{2}{|l|}{ Hypertension, n (\%) } & $11(91.7)$ & $153(79.7)$ & $30(76.9)$ & 0.755 & $53(84.1)$ & $27(87.1)$ & 1.0 \\
\hline \multicolumn{2}{|c|}{ Thyroid disease, n (\%) } & $1(8.3)$ & $23(12)$ & $8(20.5)$ & 0.116 & $11(17.5)$ & $11(35.5)$ & 0.15 \\
\hline \multicolumn{2}{|c|}{ Preoperative AF, n (\%) } & $12(100)$ & $93(48.4)$ & $19(48.7)$ & $\begin{array}{l}0.001^{\mathrm{a}} \\
0.002^{\mathrm{c}}\end{array}$ & $31(49.2)$ & $25(80.6)$ & 0.007 \\
\hline \multicolumn{2}{|c|}{$\begin{array}{l}\text { Preoperative pacemaker } \\
\text { implantation, n (\%) }\end{array}$} & $0(0)$ & $6(3.1)$ & $2(5.1)$ & 0.336 & $6(9.5)$ & $5(16.1)$ & 0.50 \\
\hline \multicolumn{2}{|l|}{ LVEF, \% } & $55(48.8-60)$ & $60(50-65)$ & $60(60-65)$ & $0.008^{a}$ & $40(30-52.5)$ & $50(40-55)$ & 0.27 \\
\hline \multicolumn{2}{|l|}{ Previous PCI, n (\%) } & $1(8.3)$ & $31(16.1)$ & $3(8.1)$ & 0.697 & $33(52.4)$ & $0(0)$ & 0.003 \\
\hline \multicolumn{2}{|l|}{ COPD, n (\%) } & $0(0)$ & $14(7.6)$ & $0(0)$ & 0.462 & $6(9.5)$ & $2(6.5)$ & 1.0 \\
\hline \multicolumn{2}{|l|}{ Renal failure, n (\%) } & $0(0)$ & $3(1.6)$ & $1(2.6)$ & 0.292 & $2(3.2)$ & $0(0)$ & 1.0 \\
\hline \multicolumn{2}{|c|}{ EuroSCORE II, points } & $2.4(1.2-3)$ & $1.4(0.9-2.6)$ & $1.2(0.8-1.9)$ & 0.063 & $3.3(2.1-6.1)$ & $2.4(1.4-3.7)$ & 0.04 \\
\hline \multicolumn{2}{|l|}{ STS score, points } & $1.6(1.1-1.9)$ & $1.2(0.6-1.9)$ & $0.8(0.5-1.4)$ & $0.004^{a}$ & $2.3(1.5-3.6)$ & $1.4(0.9-2.5)$ & 0.005 \\
\hline
\end{tabular}

Data are presented as median (interquartile range) unless otherwise indicated.

a Rheumatic heart disease vs Barlow disease;

b Fibroelastic deficiency vs Barlow disease;

c Rheumatic heart disease vs fibroelastic deficiency

Abbreviations: see TABLE 1

( $P=0.04, P=0.006$, and $P<0.001$, respectively). The higher incidence of all these risk factors in the secondary MR group resulted in more patients being assessed as high risk. Moreover, the EuroSCORE II and STS score indexes were much higher in these patients $(P<0.001$ for both comparisons) (TABLE1).

In the primary MR group, the most common etiological factor was fibroelastic deficiency (79\%), followed by Barlow disease (16\%) and rheumatic etiology $(5 \%)(P<0.001)$. Patients with Barlow disease were younger (median age, 61 years [IQR, 47-67]) than those with fibroelastic deficiency and rheumatic disease (median age, 65 years [IQR, 60-70] and 68.5 years [IQR, 64.870]; $P=0.001$ and $P=0.003$, respectively). Preoperative atrial fibrillation occurred more often in patients with rheumatic disease as compared with those with fibroelastic deficiency and Barlow disease $(P=0.002$ and $P=0.001$, respectively). Patients with rheumatic heart disease also had lower LVEF and higher STS score compared with patients with Barlow disease $(P=0.008$ and $P=0.004$, respectively) (TABLE2).

Secondary MR was seen in $67 \%$ of patients with ischemic heart disease and $33 \%$ of those with dilated cardiomyopathy $(P<0.001)$. Patients with ischemic cardiomyopathy had higher incidence of severe heart failure symptoms and percutaneous coronary interventions than patients with dilated cardiomyopathy $(P=0.02$ and $P=0.003$, respectively). Preoperative atrial fibrillation occurred more frequently in the latter group $(P=0.007)$. The EuroSCORE II and STS score were higher in the ischemic group than in the dilated cardiomyopathy group $(P=0.04$ and $P=0.005$, respectively) (TABLE2).

Perioperative characteristics Mitral valve repair or replacement with concomitant CABG was preformed more often in patients with ischemic MR, whereas MV repair alone was performed much more frequently in the primary MR group ( $P<0.001$ for all comparisons) (TABLE 3 ). 
TABLE 3 Perioperative characteristics comparing patients with primary and secondary mitral regurgitation

\begin{tabular}{|c|c|c|c|c|c|}
\hline \multicolumn{2}{|l|}{ Variable } & $\begin{array}{l}\text { All patients } \\
(n=337)\end{array}$ & $\begin{array}{l}\text { Primary MR } \\
(n=243)\end{array}$ & $\begin{array}{l}\text { Secondary MR } \\
(n=94)\end{array}$ & $P$ value \\
\hline \multicolumn{2}{|c|}{ Aortic cross-clamp time, min } & $79.5(62-100)$ & $80(61.2-104.8)$ & $79(62.2-95)$ & 0.41 \\
\hline \multicolumn{2}{|c|}{ Cardiopulmonary bypass time, min } & $124.5(99.5-164)$ & $121(98-160)$ & $127.5(102.2-170)$ & 0.16 \\
\hline \multirow[t]{7}{*}{ Type of procedure, $\mathrm{n}(\%)$} & MV repair & $131(38.9)$ & $105(43.2)$ & $26(27.7)$ & \multirow[t]{7}{*}{$<0.001$} \\
\hline & MV repair + CABG & $44(13)$ & $16(6.6)$ & $28(29.8)$ & \\
\hline & $\begin{array}{l}\text { MV repair + other valve } \\
\text { surgery }\end{array}$ & $17(5)$ & $10(4.1)$ & $7(7.4)$ & \\
\hline & MV replacement & $72(21.4)$ & $56(23)$ & $16(17)$ & \\
\hline & MV replacement + CABG & $17(5)$ & $8(3.3)$ & $9(9.6)$ & \\
\hline & $\begin{array}{l}\text { MV replacement + other valve } \\
\text { surgery }\end{array}$ & $35(10.4)$ & $27(11.1)$ & $8(8.5)$ & \\
\hline & Harpoon MV repair & $21(6.2)$ & $21(8.6)$ & $0(0)$ & \\
\hline \multicolumn{2}{|c|}{ Transfusion (red cells/platelets/plasma), units } & $2(0-7)$ & $2(0-6)$ & $3(1-7)$ & 0.10 \\
\hline \multicolumn{2}{|c|}{ Postoperative drainage $>800 \mathrm{ml}, \mathrm{n}(\%)$} & $74(21.9)$ & $48(19.8)$ & $26(27.7)$ & 0.15 \\
\hline \multicolumn{2}{|c|}{ Rethoracotomy for bleeding, $\mathrm{n}(\%)$} & $17(5)$ & $10(4.1)$ & $7(7.4)$ & 0.26 \\
\hline \multicolumn{2}{|l|}{ Low cardiac output, n (\%) } & $53(15.7)$ & $34(14)$ & $19(20.2)$ & 0.21 \\
\hline \multicolumn{2}{|c|}{ Postoperative myocardial infarction, $\mathrm{n}(\%)$} & $7(2.1)$ & $4(1.6)$ & $3(3.2)$ & 0.40 \\
\hline \multicolumn{2}{|c|}{ Intubation time $>24 \mathrm{~h}, \mathrm{n}(\%)$} & $20(5.9)$ & $15(6.2)$ & $5(5.3)$ & 0.97 \\
\hline \multicolumn{2}{|c|}{ New postoperative $A F, n(\%)$} & $19(5.6)$ & $14(5.8)$ & $5(5.3)$ & 1.0 \\
\hline \multicolumn{2}{|c|}{ Permanent pacemaker implantation following surgery, $\mathrm{n}(\%)$} & $22(6.5)$ & $16(6.6)$ & $6(6.4)$ & 1.0 \\
\hline \multicolumn{2}{|l|}{ Wound infection, $\mathrm{n}(\%)$} & $13(3.8)$ & $11(4.5)$ & $2(2.1)$ & 0.53 \\
\hline \multicolumn{2}{|l|}{ Hospital length of stay, $d$} & $11(9-16)$ & $11(9-16)$ & $11(9-16)$ & 0.66 \\
\hline \multicolumn{2}{|l|}{ In-hospital death, $\mathrm{n}(\%)$} & $11(3.3)$ & $9(3.7)$ & $2(2.1)$ & 0.13 \\
\hline
\end{tabular}

Data are presented as median (interquartile range) unless otherwise indicated.

a Overall $P$ value

Abbreviations: CABG, coronary artery bypass grafting; MV, mitral valve; others, see TABLE 1

In patients with rheumatic disease, $\mathrm{MV}$ repair was performed much less often than in patients with fibroelastic deficiency and Barlow disease, while MV replacement with concomitant other valve surgery was performed more frequently in rheumatic patients $(P=0.01$ and $P=0.04$, respectively). All Harpoon procedures were performed in patients with fibroelastic deficiency $(P<0.001)$. Among the secondary MR group, $\mathrm{MV}$ repair alone was more common in patients with dilated cardiomyopathy compared with patients with ischemic heart disease $(P=0.01)$ (TABLE4).

Of the 337 patients, 53 patients $(15.7 \%)$ developed LCO (TABLE 3). There were no differences in the incidence of LCO between groups. $\mathrm{Pa}$ tients with LCO were older, had higher occurrence of severe heart failure symptoms, impaired LVEF, and renal insufficiency $(P=0.02, P=0.006$, $P=0.01$, and $P=0.05$, respectively). Also the EuroSCORE II and STS score were higher in this group ( $P=0.006$ and $P=0.002$, respectively). We observed LCO much more often in patients after concomitant other valve surgery $(P=0.04)$. Patients with LCO syndrome also had a higher frequency of new-onset postoperative atrial fibrillation, wound infections, and blood transfusion therapy $(P=0.004, P=0.008$, and $P<0.001$, respectively) (data not shown).

The incidence of other common complications following cardiac surgery (new postoperative atrial fibrillation, postoperative drainage, postoperative myocardial infarction, intubation time, permanent pacemaker following surgery, wound infections, and transfusion therapy) also did not differ between groups. Only the length of hospital stay was greater in patients with dilated cardiomyopathy compared with patients with ischemic heart disease ( $P=0.04$ ) (tABles3 and 4 ).

In-hospital death In the early postoperative period, 11 patients (3.3\%) died, including 3.7\% of patients from the primary MR group and $2.1 \%$ from the secondary MR group $(P=0.13)$. There were no differences between the subgroups 
W TABLE 4 Perioperative characteristics of patients according to etiology of mitral regurgitation

\begin{tabular}{|c|c|c|c|c|c|c|c|c|}
\hline \multirow{2}{*}{\multicolumn{2}{|c|}{ Variable }} & \multicolumn{3}{|c|}{ Primary MR $(n=243)$} & \multirow[t]{2}{*}{$P$ value } & \multicolumn{2}{|c|}{ Secondary MR $(n=94)$} & \multirow[t]{2}{*}{$P$ value } \\
\hline & & $\begin{array}{l}\text { Rheumatic heart disease } \\
\quad(n=12)\end{array}$ & $\begin{array}{l}\text { Fibroelastic deficiency } \\
\qquad(n=192)\end{array}$ & $\begin{array}{l}\text { Barlow disease } \\
(\mathrm{n}=39)\end{array}$ & & $\begin{array}{l}\text { Ischemic heart disease } \\
\quad(n=63)\end{array}$ & $\begin{array}{l}\text { Dilated cardiomyopathy } \\
(n=31)\end{array}$ & \\
\hline \multicolumn{2}{|c|}{ Aortic cross-clamp time, min } & $60.5(57.8-76.5)$ & $81.5(63.8-104.2)$ & $77(60-110)$ & 0.07 & $87(68.5-97)$ & $65(46.5-81.5)$ & 0.002 \\
\hline \multicolumn{2}{|c|}{ Cardiopulmonary bypass time, min } & $113(80-129.2)$ & $124(100-159)$ & $110(90-171)$ & 0.10 & $137(108-178)$ & $120(94.5-149)$ & 0.09 \\
\hline \multirow[t]{7}{*}{ Type of procedure, n (\%) } & MV repair & $2(16.7)$ & $88(45.8)$ & $15(38.5)$ & \multirow[t]{7}{*}{$<0.001^{\mathrm{a}}$} & $12(19)$ & $14(45.2)$ & \multirow[t]{7}{*}{$0.004^{a}$} \\
\hline & MV repair + CABG & $0(0)$ & $14(7.3)$ & $2(5.1)$ & & $24(38.1)$ & $4(12.9)$ & \\
\hline & MV repair + other valve surgery & $0(0)$ & $8(4.2)$ & $2(5.1)$ & & $4(6.3)$ & $3(9.7)$ & \\
\hline & MV replacement & $4(33.3)$ & $36(18.7)$ & $16(41.0)$ & & $10(15.9)$ & $6(19.4)$ & \\
\hline & MV replacement + CABG & $1(8.3)$ & $6(3.1)$ & $1(2.6)$ & & $9(14.3)$ & $0(0)$ & \\
\hline & MV replacement + other valve surgery & $5(41.7)$ & $19(9.9)$ & $3(7.7)$ & & $4(6.3)$ & $4(12.9)$ & \\
\hline & Harpoon MV repair & $0(0)$ & $21(10.9)$ & $0(0)$ & & $0(0)$ & $0(0)$ & \\
\hline \multicolumn{2}{|c|}{ Transfusion (red cells/platelets/plasma), units } & $2.0(1.8-5.2)$ & $2(0-6)$ & $3(0-6)$ & 0.52 & $4.0(1-9)$ & $2(0.5-4.5)$ & 0.18 \\
\hline \multicolumn{2}{|c|}{ Postoperative drainage >800 ml, $\mathrm{n}(\%)$} & $3(25)$ & $34(17.7)$ & $11(28.2)$ & 0.13 & $20(31.7)$ & $6(19.4)$ & 0.31 \\
\hline \multicolumn{2}{|c|}{ Rethoracotomy for bleeding, n (\%) } & $2(16.7)$ & $6(3.1)$ & $2(5.1)$ & 0.25 & $6(9.5)$ & $1(3.2)$ & 0.42 \\
\hline \multicolumn{2}{|l|}{ Low cardiac output, n (\%) } & $3(25)$ & $24(12.5)$ & $7(17.9)$ & 0.18 & $13(20.6)$ & $6(19.4)$ & 1.0 \\
\hline \multicolumn{2}{|c|}{ Postoperative myocardial infarction, n (\%) } & $0(0)$ & $2(1)$ & $2(5.1)$ & 0.13 & $2(3.2)$ & $1(3.2)$ & 1.0 \\
\hline \multicolumn{2}{|c|}{ Intubation time >24 h, n (\%) } & $0(0)$ & $14(7.3)$ & $1(2.6)$ & 0.47 & $4(6.3)$ & $1(3.2)$ & 0.89 \\
\hline \multicolumn{2}{|c|}{ New postoperative AF, n (\%) } & $0(0)$ & $12(6.2)$ & $2(5.1)$ & 0.99 & $4(6.3)$ & $1(3.2)$ & 0.99 \\
\hline \multicolumn{2}{|c|}{ Permanent pacemaker implantation following surgery, $\mathrm{n}(\%)$} & $1(8.3)$ & $12(6.2)$ & $3(7.7)$ & 0.57 & $2(3.2)$ & $4(12.9)$ & 0.09 \\
\hline \multicolumn{2}{|l|}{ Wound infection, $\mathrm{n}(\%)$} & $0(0)$ & $9(4.7)$ & $2(5.1)$ & 0.16 & $2(3.2)$ & $0(0)$ & 1.000 \\
\hline \multicolumn{2}{|l|}{ Hospital length of stay, $d$} & $10(6.8-11.8)$ & $11(9-17)$ & $10(9-13)$ & 0.12 & $10(8-14.5)$ & $13(10-16.5)$ & 0.04 \\
\hline \multicolumn{2}{|l|}{ In-hospital death, n (\%) } & $1(8.3)$ & $7(3.6)$ & $1(2.6)$ & 0.43 & $1(1.6)$ & $1(3.2)$ & 0.26 \\
\hline
\end{tabular}

Data are presented as median (interquartile range) unless otherwise indicated.

a Overall $P$ value

Abbreviations: see TABLES 1 and 3 
regarding etiological factor of $\mathrm{MR}(P=0.13)$ (TABLE 3 and 4). Univariate analysis revealed that the higher risk of death was associated with older age, severe heart failure symptoms, impaired LVEF, previous percutaneous coronary interventions, $\mathrm{CPB}$ time, $\mathrm{LCO}$, and wound infections $(P=0.004, P<0.001, P=0.005, P=0.009$, $P=0.002, P=0.006$, and $P=0.03$, respectively). Also MV replacement with concomitant other valve surgery increased the risk of mortality $(P=0.049)$ (table 5).

DISCUSSION In this study, we have shown the distribution of MR etiology and evaluated the clinical outcomes and risk factors in predicting death in patients undergoing mitral surgery for different types of MR. We found that the etiological mechanism of MR did not correlate with the postoperative clinical course as well as with the hospital mortality rate. In-hospital mortality correlated with the type of procedure and associated comorbidities rather than with the etiological mechanism of MR.

The definition of severe MR involving cutoff points for effective regurgitant orifice area and regurgitant volume (quantitative parameters) is widely discussed. According to the European Society of Cardiology and European Association for Cardio-Thoracic Surgery guidelines, the cutoff points for effective regurgitant orifice area and regurgitant volume for severe primary $M R$ are $0.4 \mathrm{~cm}^{2}$ and $60 \mathrm{ml}$, and for severe secondary $\mathrm{MR}, 0.2 \mathrm{~cm}^{2}$ and $30 \mathrm{ml}$, respectively. There is a noticeable trend towards performing the MV surgery in secondary MR with the same quantitative parameters as in primary MR, thus coming closer to the American College of Cardiology and American Heart Association guidelines. According to these guidelines the recommended definition of severe secondary MR is now the same as of primary MR. ${ }^{14}$

It is crucial to integrate the clinical and echocardiographic findings to make a good decision for patients, especially in secondary MR. Making an appropriate estimation of the etiology and underlying MR mechanism is a crucial part of adequate management. ${ }^{7}$ The major cause of MR in this study group was primary $\mathrm{MR}$, occurring in about $70 \%$ of patients, which is consistent with the results of other studies, where the degeneration of the MV apparatus was also the most common etiological factor of MR. ${ }^{14,15}$ The European Registry of MR showed a more balanced frequency of primary and secondary MR. ${ }^{8}$ This discrepancy might result from distinguishing mixed types of MR in the registry, described as concomitant presence of both mechanisms of MR. Furthermore, we classified etiologies of MR not only on the basis of echocardiographic criteria but also surgical findings. In the Euro Heart Survey study, where surgical cases were also included, primary forms of MR constituted a proportion similar to our results. ${ }^{16}$

In this study, among patients with primary $\mathrm{MR}$, the most common causative factor was fibroelastic deficiency, followed by Barlow disease and rheumatic etiology. Although the European Registry of MR was not a population-based epidemiological study and did not report the prevalence of MR in the general population, the results regarding the etiology of primary MR are consistent with our outcomes. ${ }^{8}$ In our setting, ischemic cardiomyopathy was the most common etiology of secondary MR (67\%), and this finding is also consistent with other results. ${ }^{8,14,16}$ The study group did not include patients with severe left ventricular dysfunction (ie, LVEF <30\%), in whom other treatment (including MitraClip technique) was used. This may be a typical scenario for the current population of patients with secondary MR, who are considered to be individuals at very high surgical risk and with a relative contraindication to open heart surgery according to the guidelines. ${ }^{1}$ The fact that we excluded patients with infective endocarditis and prosthetic valve dysfunction also deserves a comment. Despite improvements in their management, infective endocarditis and prosthetic valve dysfunction remain associated with high mortality and severe complications. Surgical therapy of the diseases is associated with perioperative risk. ${ }^{17,18}$ In our institution, surgery was usually performed in this group of patients on an emergency or urgent basis, irrespective of the duration of antibiotic treatment, which further increased the operative risk scores. We excluded these patients from the study to minimize differences between groups.

Although the EuroSCORE II and STS score were much higher in patients with secondary $\mathrm{MR}$, the postoperative course and in-hospital mortality did not differ compared with those in patients with primary MR. Also, no differences in terms of postoperative outcomes and death were noted for different types of primary as well as secondary MR mechanisms. This study indicates that the hospital death rate correlated with the type of procedure and associated comorbidities rather than the etiological mechanism of MR. Mitral valve replacement with concomitant other valve surgery increased the risk of postoperative death. According to recent studies assessing early results of multiple valve operations, in-hospital mortality rates range from $2.5 \%$ to $20 \%{ }^{16,19}$ In our study, this group of patients had the highest in-hospital mortality rate, that is, $14.3 \%$, and these results are in line with current literature. ${ }^{20,21}$ In contrast to our results, Mkalaluh et $\mathrm{al}^{22}$ determined CABG as the only concomitant procedure that was a predictor of early mortality after MV surgery. The discrepancy might result from different 


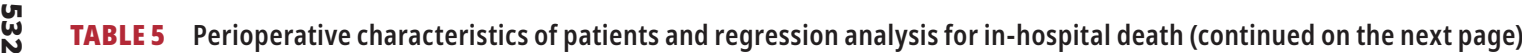

\begin{tabular}{|c|c|c|c|c|c|}
\hline Variable & & All patients $(n=337)$ & Survivors $(n=326)$ & In-hospital death $(n=11)$ & $P$ value \\
\hline Age, $y$ & & $65(60.5-75)$ & $65(59-71)$ & $71(66-74.5)$ & 0.004 \\
\hline Male, n (\%) & & $224(66.5)$ & $217(66.6)$ & $7(63.6)$ & 0.06 \\
\hline \multirow[t]{2}{*}{ Type of MR, n (\%) } & Primary & $243(72.1)$ & $234(71.8)$ & $9(81.8)$ & \multirow[t]{2}{*}{0.13} \\
\hline & Secondary & $94(27.9)$ & $92(28.2)$ & $2(18.2)$ & \\
\hline \multirow[t]{5}{*}{ Mechanism of MR, $\mathrm{n}(\%)$} & Rheumatic heart disease & $12(3.6)$ & $11(3.4)$ & $1(9.1)$ & \multirow[t]{5}{*}{0.13} \\
\hline & Fibroelastic deficiency & $192(57)$ & $185(56.7)$ & $7(63.6)$ & \\
\hline & Barlow disease & $39(11.6)$ & $38(11.6)$ & $1(9.1)$ & \\
\hline & Ischemic heart disease & $63(18.7)$ & $62(19)$ & $1(9.1)$ & \\
\hline & Dilated cardiomyopathy & $31(9.2)$ & $30(9.2)$ & $1(9.1)$ & \\
\hline $\mathrm{BSA}, \mathrm{m}^{2}$ & & $1.9(1.7-2)$ & $1.9(1.8-2)$ & $1.8(1.6-1.9)$ & 0.06 \\
\hline $\mathrm{BMI}, \mathrm{kg} / \mathrm{m}^{2}$ & & $26.4(24.2-29.6)$ & $26.6(24.2-29.6)$ & $25.6(23.4-28.4)$ & 0.32 \\
\hline \multirow[t]{2}{*}{ NYHA, n (\%) } & I/II & $206(61.1)$ & $204(62.6)$ & $2(18.2)$ & \multirow[t]{2}{*}{$<0.001$} \\
\hline & III/IV & $131(38.9)$ & $122(37.4)$ & $9(81.2)$ & \\
\hline Previous stroke, $\mathrm{n}(\%)$ & & $32(9.5)$ & $31(9.5)$ & $1(9.1)$ & 0.11 \\
\hline Diabetes mellitus, n (\%) & & $64(19)$ & $60(18.4)$ & $4(36.4)$ & 0.08 \\
\hline Hypertension, n (\%) & & $274(81.3)$ & $264(81)$ & $10(90.1)$ & 0.39 \\
\hline Thyroid disease, $\mathrm{n}(\%)$ & & $54(16)$ & $53(16.2)$ & $1(9.1)$ & 0.25 \\
\hline Preoperative AF, $\mathrm{n}(\%)$ & & $180(53.4)$ & $172(52.8)$ & $8(72.7)$ & 0.08 \\
\hline Preoperative pacemaker implantation, $n(\%)$ & & $19(5.6)$ & $18(5.5)$ & $1(9.1)$ & 0.31 \\
\hline LVEF, \% & & $58(46-65)$ & $60(48-65)$ & $50(33.8-52.5)$ & 0.005 \\
\hline Previous PCI, n (\%) & & $68(20.2)$ & $63(19.3)$ & $5(45.4)$ & 0.009 \\
\hline COPD, n (\%) & & $22(6.5)$ & $21(6.4)$ & $1(9.1)$ & 0.38 \\
\hline Renal failure, $n(\%)$ & & $6(1.8)$ & $5(1.5)$ & $1(9.1)$ & 0.31 \\
\hline EuroSCORE II, points & & $1.7(1-3.3)$ & $1.6(1-3.1)$ & $3.5(2.6-13.7)$ & $<0.001$ \\
\hline STS score, points & & $1.4(0.7-2.4)$ & $1.3(0.7-2.2)$ & $2.6(1.6-5.5)$ & $<0.001$ \\
\hline Aortic cross-clamp time, min & & $79.5(62-100)$ & $80(61-100)$ & $79(74-96)$ & 0.39 \\
\hline Cardiopulmonary bypass time, min & & $124.5(99.5-164)$ & $120(98.8-160)$ & $162(132-191.5)$ & 0.002 \\
\hline
\end{tabular}


TABLE 5 Perioperative characteristics of patients and regression analysis for in-hospital death (continued from the previous page)

\section{Variable}

Type of procedure, $\mathrm{n}(\%)$
All patients $(n=337)$

MV repair

$M V$ repair + CABG

MV repair + other valve surgery

MV replacement

MV replacement + CABG

MV replacement + other valve surgery

Harpoon MV repair

Transfusion (red cells/platelets/plasma), units

Postoperative drainage $>800 \mathrm{ml}, \mathrm{n}(\%)$

Rethoracotomy for bleeding, $\mathrm{n}(\%)$

Low cardiac output, $n(\%)$

Postoperative myocardial infarction, $\mathrm{n}(\%)$

Intubation time $>24 \mathrm{~h}, \mathrm{n}(\%)$

New postoperative $A F, n(\%)$

Permanent pacemaker implantation following surgery, $n(\%)$

Wound infection, $\mathrm{n}(\%)$

Hospital length of stay, $d$

Data are presented as median (interquartile range) unless otherwise indicated.

a Overall $P$ value

Abbreviations: see TABLES 1 and 3

$17(5)$

$17(5)$

$35(10.4)$

$21(6.2)$

$17(5)$

$53(15.7)$

$7(2.1)$

$20(5.9)$

$19(5.6)$

$13(3.8)$

$11(9-16)$

Survivors $(n=326)$

In-hospital death $(n=11)$

$P$ value

$131(38.9) \quad 128(39.3)$

$128(39.3)$

$3(27.3)$

$43(13.2)$

$1(9.1)$

$17(5.2)$

$0(0)$

$72(21.4) \quad 71(21.8) \quad 1(9.1)$

$16(4.9)$

$1(9.1)$

$31(9.5)$

$20(6.1)$

$2(0-7) \quad 2(0-6)$

$74(21.9) \quad 71(21.8)$

$71(21.8)$

$16(4.9)$

48 (14.7)

5 (1.5)

$19(5.8)$

18 (5.5)

$22(6.7)$

$11(3.4)$

$11(9-16)$

4 (36.4)

$1(9.1)$

$3(27.3)$

$1(9.1)$

$5(45.4)$

$2(18.2)$

1 (9.1)

1 (9.1)

$0(0)$

2 (18.2)

14 (3-29) 
patient characteristics, as Mkalaluh et $\mathrm{al}^{22}$ analyzed only octogenarians, and patients in our study were much younger.

In this study, besides concomitant other valve surgery, other factors associated with higher risk of death were age, severe heart failure symptoms, impaired LVEF, previous percutaneous coronary interventions, CPB time, LCO, and wound infections. Our results are in line with the current literature. In an attempt to identify independent risk factors for perioperative mortality, Akay et $\mathrm{al}^{23}$ found that preoperative severe heart failure symptoms, reduced LVEF, and increased left ventricular end-diastolic diameter were factors associated with increased mortality in MV surgery. In a study by Lio et al, ${ }^{17}$ previous cardiac interventions, preoperative LVEF, prolonged cardiopulmonary bypass, and postoperative complications were factors that impacted short-term survival. Similarly, Pagni et a $1^{18}$ showed that advanced age and LCO syndrome were also associated with increased perioperative risk.

Several limitations of our study should be acknowledged. It was a single-center study on a relatively small population with multiple combinations of procedures and different cardiac operators. The groups included into the analysis differed in age, symptom severity, comorbidities, and LVEF. The presence of these cofounding factors hampers the analysis of the impact of the etiology on surgical outcomes. On the other hand, the study was performed in real-life setting of a cardiosurgical unit and enrolled unselected consecutive patients. The low incidence of the clinical endpoints may have impacted the strength of this study and may have biased the outcomes. No multivariate logistic regression analysis was performed because of a low number of clinical endpoint events. Finally, our study was limited to the hospital stay. It would be of interest to assess long-term follow-up.

In conclusion, this study indicates that the clinical outcomes including in-hospital mortality correlated with the type of procedure and concomitant perioperative comorbidities rather than with the etiological mechanism of MR itself.

\section{ARTICLE INFORMATION}

CONFLICT OF INTEREST None declared.

HOW TO CITE Rzucidło-Resil J, Plicner D, Gackowski A, et al. The impact of the mechanism of mitral regurgitation on clinical outcomes in patients after mitral valve surgery. Kardiol Pol. 2019; 77: 525-534. doi:10.5603/KP.a2019.0043

\section{REFERENCES}

1 Baumgartner H, Falk V, Bax IJ, et al. 2017 ESC/EACTS Guidelines for the management of valvular heart disease. Eur Heart J. 2017; 38: 2739-2791.

2 Iung B, Baron G, Tornos P, et al. Valvular heart disease in the community: a European experience. Curr Probl Cardiol. 2007; 32: 609-661.

3 Praet KMV, Stamm C, Sündermann SH, et al. Minimally invasive surgical mitral valve repair: state of the art review. Interv Cardiol. 2018; 13: 14-19.

4 Tolis G Jr, Sundt TM 3rd. Surgical strategies for management of mitral regurgitation: recent evidence from randomized controlled trials. Curr Atheroscler Rep. 2015; 17: 67-71.
5 Shuhaiber J, Anderson RJ. Meta-analysis of clinical outcomes following surgical mitral valve repair or replacement. Eur J Cardiothorac Surg. 2007; 31: 267-275. 6 Russell EA, Walsh WF, Reid CM, et al. Outcomes after mitral valve surgery for rheumatic heart disease. Heart Asia. 2017; 9: 1-7.

7 Madesis A, Tsakiridis K, Zarogoulidis $P$, et al. Review of mitral valve insufficiency: repair or replacement. J Thorac Dis. 2014; 6: 39-51.

8 Monteagudo Ruiz JM, Galderisi M, Buonauro A, et al. Overview of mitral regurgitation in Europe: results from the European Registry of mitral regurgitation (EuMiClip). Eur Heart J Cardiovasc Imaging. 2018; 19: 503-507.

9 Flachskampf FA, Wouters PF, Edvardsen T, et al. Recommendations for transoesophageal echocardiography: EACVI update 2014. Eur Heart J Cardiovasc Imaging. 2014; 15: 353-365.

10 Algarni KD, Maganti M, Yau TM. Predictors of low cardiac output syndrome after isolated coronary artery bypass surgery: trends over 20 years. Ann Thorac Surg. 2011; 92: 1678-1684.

11 Lomivorotov WV, Efremov SM, Kirov MY, et al. Low-cardiac-output syndrome after cardiac surgery. J Cardiothorac Vasc Anesth. 2017; 31: 291-308.

12 Shen J, Lall S, Zheng V, et al. The persistent problem of new onset postoperative atrial fibrillation: A single institution experience over two decades. J Thorac Cardiovasc Surg. 2011; 141: 559-570.

13 Gammie JS, Wilson P, Bartus K, et al. Transapical beating-heart mitral valve repair with an expanded polytetrafluoroethylene cordal implantation device: initial clinical experience. Circulation. 2016; 134: 189-197.

14 Mick SL, Keshavamurthy S, Gillinov AM. Mitral valve repair versus replacement. Ann Cardiothorac Surg. 2015; 4: 230-237.

15 Glauber M, Miceli A, Canarutto D, et al. Early and long-term outcomes of minimally invasive mitral valve surgery through right minithoracotomy: a 10-year experience in 1604 patients. J Cardiothorac Surg. 2015; 10: 181-189.

16 Iung B, Baron G, Butchart EG, et al. A prospective survey of patients with valvular heart disease in Europe: the Euro Heart Survey on Valvular Heart Disease. Eur Heart J 2003; 24: 1231-1243.

17 Habib G, Lancellotti P, Antunes MJ, et al. ESC Scientific Document Group. 2015 ESC Guidelines for the management of infective endocarditis: The Task Force for the Management of Infective Endocarditis of the European Society of Cardiology (ESC). Endorsed by: European Association for Cardio-Thoracic Surgery (EACTS), the European Association of Nuclear Medicine (EANM). Eur Heart J. 2015; 36: 3075-3128.

18 Ma WG, Hou B, Abdurusul A, et al. Dysfunction of mechanical heart valve prosthesis: experience with surgical management in 48 patients. J Thorac Dis. 2015; 7: 2321-2329

19 Lio A, Murzi M, Di Stefano G, et al. Triple valve surgery in the modern era: short- and long-term results from a single centre. Interact Cardiovasc Thorac Surg. 2014; 19: 978-984.

20 Pagni S, Ganzel BL, Singh R, et al. Clinical outcome after triple-valve operations in the modern era: are elderly patients at increased surgical risk? Ann Thorac Surg. 2014; 97: 569-576.

21 Gravel GM, Bouchard D, Perrault LP, et al. Triple valve surgery: clinical results of a three-decade experience. J Heart Valve Dis. 2011; 20: 75-82.

22 Mkalaluh S, Szczechowicz M, Dib B, et al. Outcomes and predictors of mortality after mitral valve surgery in high-risk elderly patients: the Heidelberg experience. Med Sci Monit. 2017; 23: 6193-6200.

23 Akay TH, Gultekin B, Ozkan S, et al. Triple-valve procedures: impact of risk factors on midterm in a rheumatic population. Ann Thorac Surg. 2006; 82: 1729-1734. 